Lymphology 53 (2020) 29-37

\title{
A MODIFIED MOUSE-TAIL LYMPHEDEMA MODEL
}

\author{
G. Arruda, S. Ariga, T.M. de Lima, H.P. Souza, M. Andrade
}

Emergency Department (LIM-51) (GA,SA,TMdL,HPS) and Department of Surgery (LIM-02) (MA), Faculdade de Medicina da Universidade de São Paulo, Brazil

\section{ABSTRACT}

One of the main obstacles to studying the pathophysiology of lymphedema development is the lack of appropriate experimental models. Following up on a mouse-tail method that has been described, we performed changes to the method which made it easier to perform in our hands and demonstrated similar results. Twenty C57Black mice were operated on using the previous technique and euthanized after 3 or 6 weeks. Another twenty mice were submitted to the new technique developed in our laboratory and euthanized at the same time points. Tissue samples were collected from the proximal part of the tail (control) and from the distal part (lymphedema) for both models. Animals in both operative groups developed marked edema in the distal part of the tail. This was characterized by lymph vessels dilation, edema, inflammatory cell infiltration, and adipose tissue deposition. Lymphedema was detected after 3 weeks in both models, reaching its maximum after 6 weeks. Adipocytes detected by histology (Oil red $O$ staining) and molecular markers for adipogenesis, lymphangiogenesis and inflammation (lipin 1 and 2, SLP76, and F4-80) were demonstrated to be increased equally in both models. In conclusion, both models provide a reliable method to study lymphedema pathophysiology. However, our modified technique is easier and faster to perform while still providing reliable and consistent results.
Keywords: lymphedema, mouse model, histology, molecular markers, signaling pathways

Lymphedema is a common clinical condition that is observed when lymphatic hypoplasia or aplasia, fibrosis, obstruction or removal of any of its components (such as lymph vessels or nodes) is present (1). There are several conditions which can primarily or secondarily lead to lymphedema, such as Milroy disease, filariasis, trauma, neoplasm invasion and lymph node dissection in cancer treatment (2). The latter has grown in importance throughout the years due to improved cancer diagnosis and therapy.

Unfortunately, lymphedema is a common complication of therapeutic surgical procedures and the use of radiotherapy to avoid local breast cancer recurrence and the reported prevalence of lymphedema in patients who undergo axillary lymph node resection following breast cancer surgical treatment varies from 7 to $77 \%$ (3). Considering the high incidence of breast cancer around the world and the disabling symptoms of lymphedema, there is an urgent need to find better treatments for this condition $(4,5)$.

Our current therapeutic arsenal is restricted, chiefly because of our lack of understanding about lymphedema pathogenesis $(6,7)$. It is clear that chronically lymphedematous limbs display tissue changes and that initial ede- 
ma progressively accumulates adipose tissue deposits $(8,9)$. The mechanisms responsible for this phenomenon, however, are not completely understood.

One of the main obstacles to studying lymphedema pathophysiology is the lack of appropriate experimental models (10). A recent systematic review compared several animal models and techniques that are used to study lymphedema (11). Authors concluded that no model is perfect with some costly and laborious to perform and others resulted in variable lymphedema duration. Most of these disadvantages are due to ethics, cost, animal mortality, level of procedural difficulty, hardships in animal captivity after procedure, variable lymphedema duration among other reasons. Nonetheless, the authors suggested mice as the best animal model due to their availability, low cost, easy captivity, ethics issues, and reproducible promising results (11).

An experimental model named mouse-tail method (12) has been utilized for this purpose, allowing the controlled evaluation of lymphatic stasis, mechanisms of lymphatic fibrosis, and regeneration as well as the consequences of lymphedema in the affected tissue (13-16). Another impact of this method is the potential to establish the molecular mechanisms by which adipose tissue accumulates using transgenic mice that could enable the study of specific signaling pathways $(13,14)$.

In this study, we describe some changes we developed in this previously described method which allow it to be performed more easily while still offering consistent results.

\section{METHODS}

\section{Ethics Committee}

This study was performed after institutional ethics committee approval for experimental mice use and project execution (CAPPESQ Protocol 84/2015).

\section{Experimental Groups}

In order to compare the development of lymphedema, we divided forty C57 Black mice in two groups.

- Model A: surgical procedures were performed according to the method proposed by Boardman and Rutkowski $(15,16)$ and modified by Clavin NW et al (12). Half of the animals in this group (10 mice) were sacrificed after 3 weeks and the other $\mathbf{1 0}$ mice were sacrificed after 6 weeks.

- Model B: surgical procedures were performed according to the technique developed in our laboratory, based on the methods described by Boardman and Rutkowski $(15,16)$ and modified by Clavin NW et al (12). Half of the animals in this group (10 mice) were sacrificed after 3 weeks and the other 10 mice were sacrificed after 6 weeks.

\section{Surgical Procedures}

All procedures were carried out by the same surgeon, followed the same technical sequence for each Model, and were performed using both disposable and aseptic material or instruments which received antiseptic treatment prior to procedure. Four animals were operated on each day with two from Model A and two from Model B.

Both methods were performed under anesthesia, which consisted of an intraperitoneal injection of ketamine $100 \mathrm{mg} / \mathrm{kg}$ and xylazine $20 \mathrm{mg} / \mathrm{kg}$ (Cristalia, Brazil).

The common procedures for both models consisted of the dissection of a 2-mm, full-thickness, circumferential segment of skin, $2 \mathrm{~cm}$ distally from the base of the tail, using microsurgical instruments and a magnifying glass for this purpose. No vascular structure is damaged during dissection. Once this segment of skin was removed and the deep structures were visible, Patent Blue V stain (Guerbet Lab, France) was injected intradermally, distal to the wound at the tip of the tail, with a $20 \mathrm{G}$ needle to allow location of the lymphatic vessels, which were typically 1 to 2 deep lymphatic vessels on each side of each lateral tail veins.

The method proposed by Boardman and 
Rutkowski $(15,16)$ and modified by Clavin NW et al (12) (reported here as Model A) involves the exclusive ligation of the deep lymphatic vessels near the lateral tail veins. In the published reports, the method to distinguish these lymphatic vessels from surrounding tissue is not clear nor is the ligation process detailed. Therefore, we ligated the lymphatic vessels using USP 10-0 nylon surgical suture.

Our proposed method is performed similarly to Model A in terms of preparation, materials, anesthesia, skin dissection, and lymphatic vessel staining using Patent Blue $\mathrm{V}$. The main difference lies on the ligation method. During the pretest and surgical training period of this research, we observed there were tiny lymphatic vessels running along the wall of the lateral veins. When one tried to ligate or dissect these vessels, either the vein wall was damaged, the procedure took longer or demanded extra anesthesia, or tail vitality was prospectively reduced. On the other hand, we also observed that, since the tail is drained by three veins which anastomose with one another, we could ligate the lateral veins together with all lymphatic vessels around them without impacting tail vitality or analyses. Additionally, this procedure demanded less time, could be taught more easily to others, and did not demand outstanding microsurgical skills. Therefore, in our proposed method (Model B), the lateral veins were ligated together with the lymphatic vessels stained by Patent Blue V using USP 5-0 nylon suture (Fig. 1). All remaining details such as bandaging, captivity, and post procedure care were also the same as Model A.

After the surgical procedure the wounds were left uncovered so that daily evaluation of lymphedema formation and tail vitality could be performed. All experimental animals were kept in individual cages and received painkiller diluted in water after the procedure until the day of analyses. Observations were performed twice daily in order to detect any discomfort and the aspect of the wounds and the tails.

After 3 weeks, half of the animals in each experimental group were sacrificed, using excess doses of anesthetics (ketamine and xylazine). The remaining animals were sacrificed 6 weeks after the procedure.

\section{Sample Preparation}

After euthanasia, animals were sacrificed and tails removed to be sectioned for histological and molecular analyses. In both groups, control tissues consisted of the area located proximal to the scar of the procedure in the tail while the lymphedema group consisted of the area distal to the scar in the tail. The proximal area of the tail $(2 \mathrm{~mm}$ proximal to the scar) was cut into two 4-mm slices. The first one was sent for histological analysis and the other one was homogenized in buffer and to Trizol for mRNA collection. The same procedure was performed on the distal part of the tail. Two similar 4-mm slices were collected for histological and molecular analyses, forming the lymphedema experimental group (Fig. 2).

\section{Histological Analyses}

For histological analyses, forty-millimeter tail sections were harvested, with the borders twenty milimeters from each side (proximal and distal) of the surgical scar. Samples were then frozen and sectioned at 10 or $15 \mu \mathrm{m}$. For observation of tissue swelling and histological changes, transverse sections of the tail were stained by hematoxylin and eosin (H\&E) on $10 \mu \mathrm{m}$ frozen sections. For observation of adipose tissue deposits, Oil red $\mathrm{O}$ staining was performed on $15 \mu \mathrm{m}$ frozen sections. Specimens were examined and compared using Leica DM2700 M Incident light microscope with coupled camera (Nikon, Japan).

\section{Quantitative Real-Time Polymerase Chain Reaction}

All RNA samples were obtained by the Trizol method (Thermo Fisher Scientific) and DNAse treatment according to producer (Thermo Fisher Scientific - \#18047019). After 


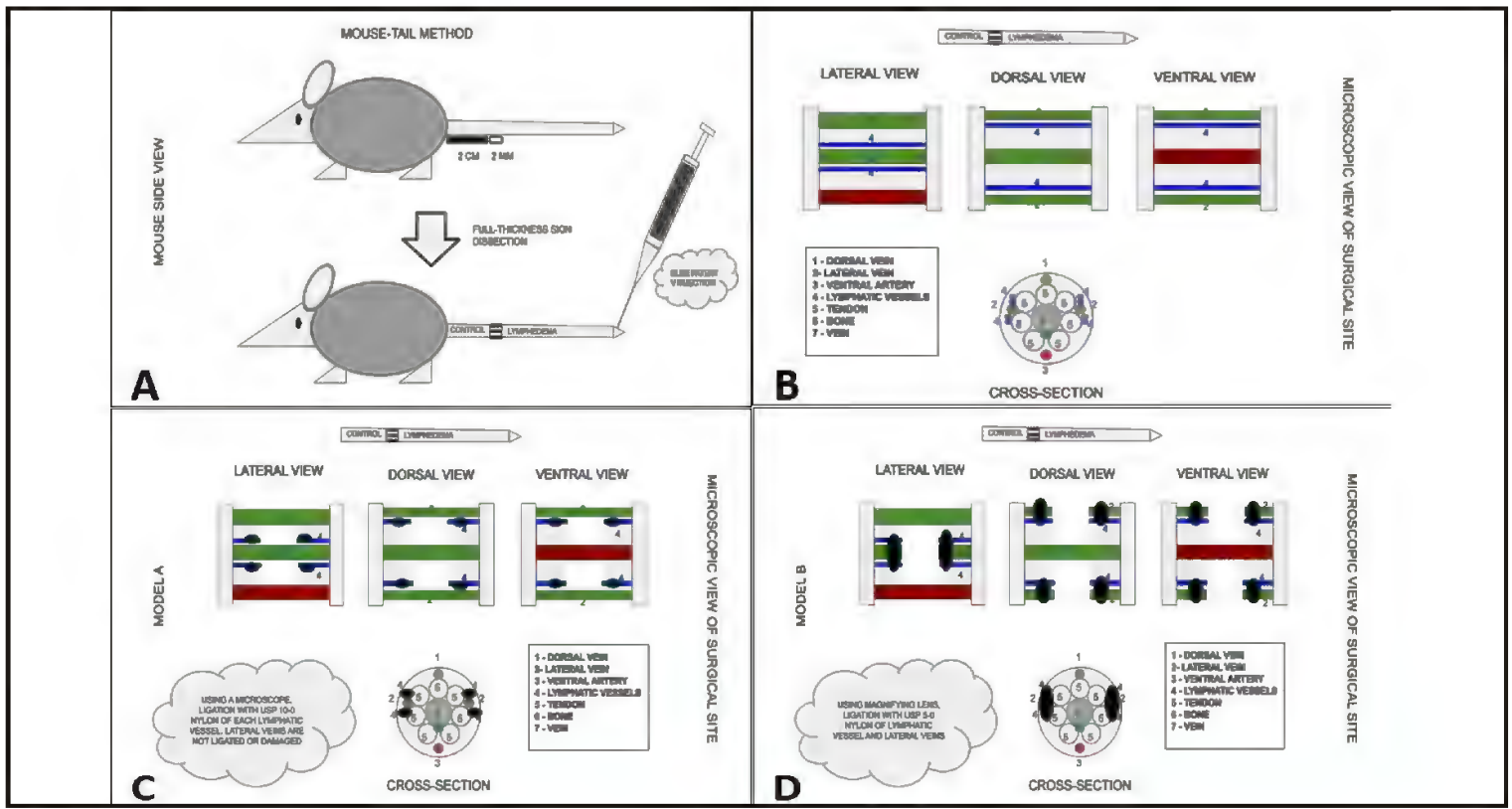

Fig.1. A) Macroscopic view of the surgical procedure in which a 2-mm full-thickness section of skin is dissected $2 \mathrm{~cm}$ from the base of the tail in order to expose the structures. In addition, this figure also displays where Blue Patent $V$ is injected to visualize the lymphatic vessels. B) Anatomic location of the veins, lymphatics, and artery in the mouse tail. C) In Model A, the lymphatic vessels are identified and individually ligated using USP 10-0 nylon suture. D) In Model B, both the lymphatic vessels and lateral vein are ligated together using USP 5-0 nylon suture.

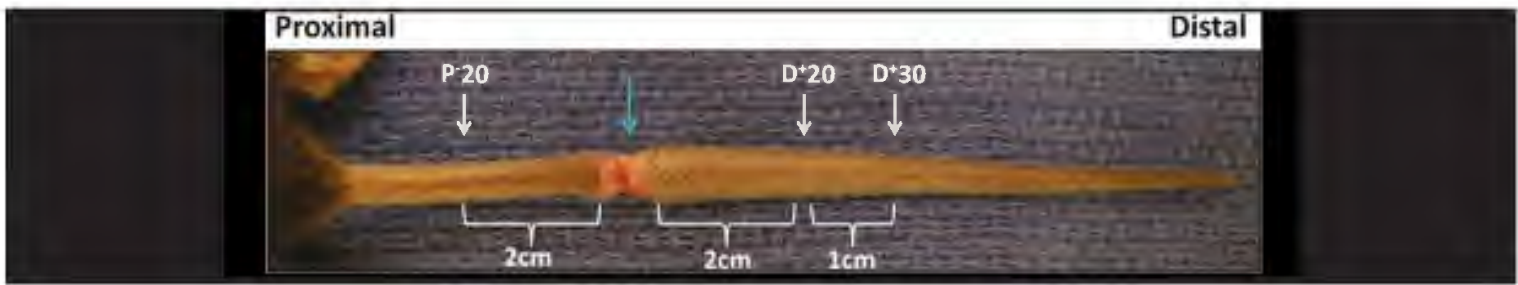

Fig.2. In both Models $A$ and B, the procedure involves the dissection of a full-thickness segment of tail skin 2-millimeters wide at 2 centimeters away from the tail base (blue arrow). After the staining of lymphatic vessels using Patent Blue V, these vessels are isolated and ligated (Model A) or ligated together with the lateral veins (Model B). This picture represents the 3-week analysis. It is possible to see the procedure scar (blue arrow). The area before the scar, proximally to the tail base, is defined as the control segment. The area after the scar, distal to the tail base, is defined lymphedema segment. It is possible to observe that the tail keeps its vitality despite the procedure and that the lymphedema segment presents clear enlargement compared to the control segment. Additionally, the dorsal vein visualized centrally in this picture maintains the venous flow regularly.

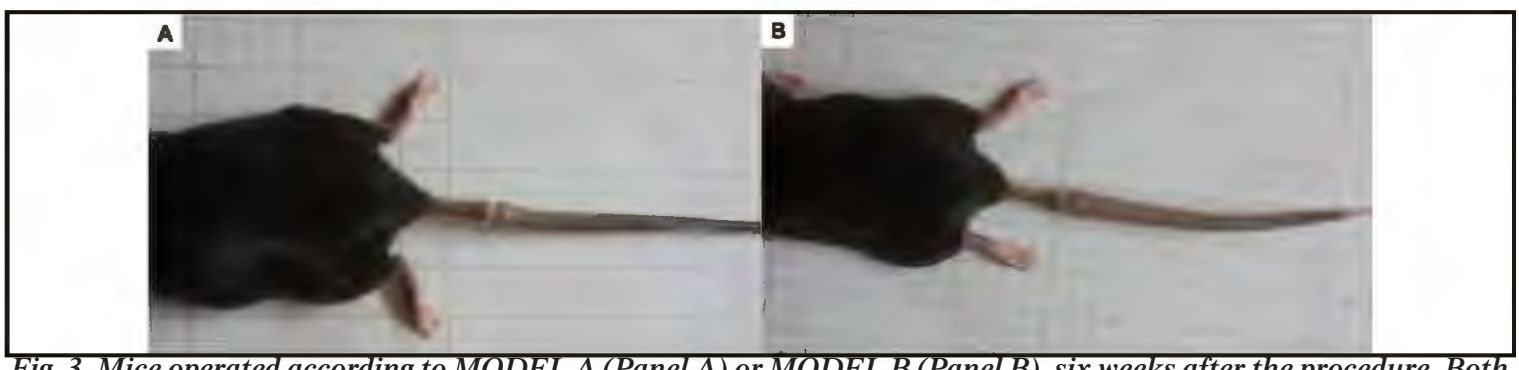

Fig. 3. Mice operated according to MODEL A (Panel A) or MODEL B (Panel B), six weeks after the procedure. Both methods led to a marked enlargement of the distal part of the tails, while the proximal part does not show significant edema, particularly farther than $20 \mathrm{~mm}$ from the scar, where the samples were collected for the control group. 
reverse transcription into cDNA, quantitative real-time polymerase chain reaction was carried out with Super Script III Platinum One-Step qRT-PCR Kit with ROX (Thermo Fisher Scientific - \#11745100) in thermocycler Step One Real-Time PCR System (Applied Biosystems) for SLP76, Lipin-1, Lipin-2, F480, and GAPDH (housekeeping gene). The extracted RNA was quantified by biophotometer (Eppendorf) in $260 \mathrm{~nm}$ wavelength. The gene expression was quantified by the CT method.

\section{Statistical Analysis}

Statistical analysis was performed using R Software. In order to compare lymphedema formation between the lymphedema and control groups in the same animal, we performed Students t-test for paired groups. To compare MODEL A to MODEL B, first we performed an F-test for Variances and, depending to the result, we performed a t-student test for similar or different variances. Results are shown as mean \pm Standard Error of the Mean. The significance level was set at $\mathbf{0 . 0 5}$ for all results.

\section{RESULTS}

\section{Morphological Changes after Surgical Proce- dure}

Animals operated on by both methods developed a marked enlargement of the distal portion of their tails. This phenomenon could be observed 3 weeks after the surgical procedure, and it was more evident after 6 weeks (Fig. 2). Moreover, no animal seemed bothered by the procedure and it did not impair their activity or feeding. Lymphedema areas were measured in animals from both groups and no difference could be observed (Figs. 3,4).

Mice operated on according to MODEL B presented no complications such as infection or tail ischemia and had no mortality. On the other hand, animals operated on according to MODEL A presented a mild tail ischemia characterized by extremity necrosis (4 out of 20 mice) but no infection or deaths. These

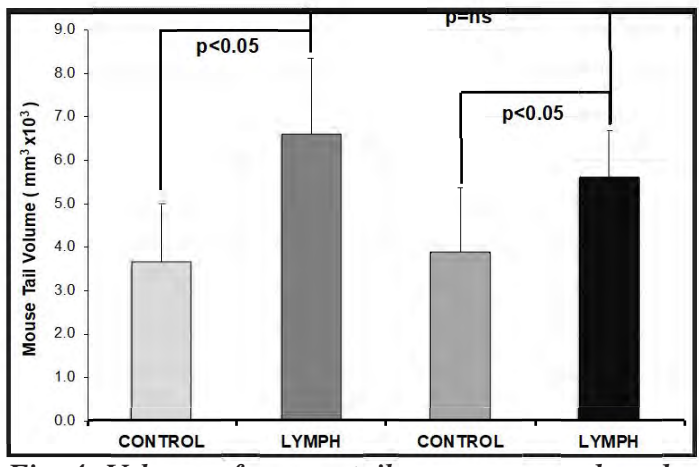

Fig. 4. Volume of mouse tails was measured as described in Methods. A marked enlargement in mouse tails was observed in the distal part of the tails (lymphedema, LYMPH group) as compared against the proximal part of the tails (CONTROL group) in both model groups. However, no difference was observed between the two models. $N=10$ in each group.

4 mice whose tails presented ischemia were excluded from analyses.

In both experimental groups, lymphedema reached the maximum volume around the sixth week and then started to regress at variable rates.

\section{Histological Analyses}

Once it was established that our model adaptation causes a similar enlargement of the distal portion of the tail, we evaluated the histological characteristics of the tissue. For both models, we compared the proximal part of the tail near the scar and where no edema was present (control) with the distal part of the tail, with marked enlargement (lymphedema). Tissues from the control group in both MODELS A and B stained with H\&E showed small amounts of adipose tissue and no dilated lymph vessels. Three weeks after the surgical procedure in the lymphedema groups, observation demonstrated enlargement of the area of fibrous sustaining tissue due to the edema and inflammatory cells infiltration particularly due to granulocytes and fibroblast proliferation. Interestingly, we also found edema around muscle fibers. It is not possible to distinguish lymphatic vessels with this staining but it is possible to visualize enlarged vessels in the edematous tissue. 


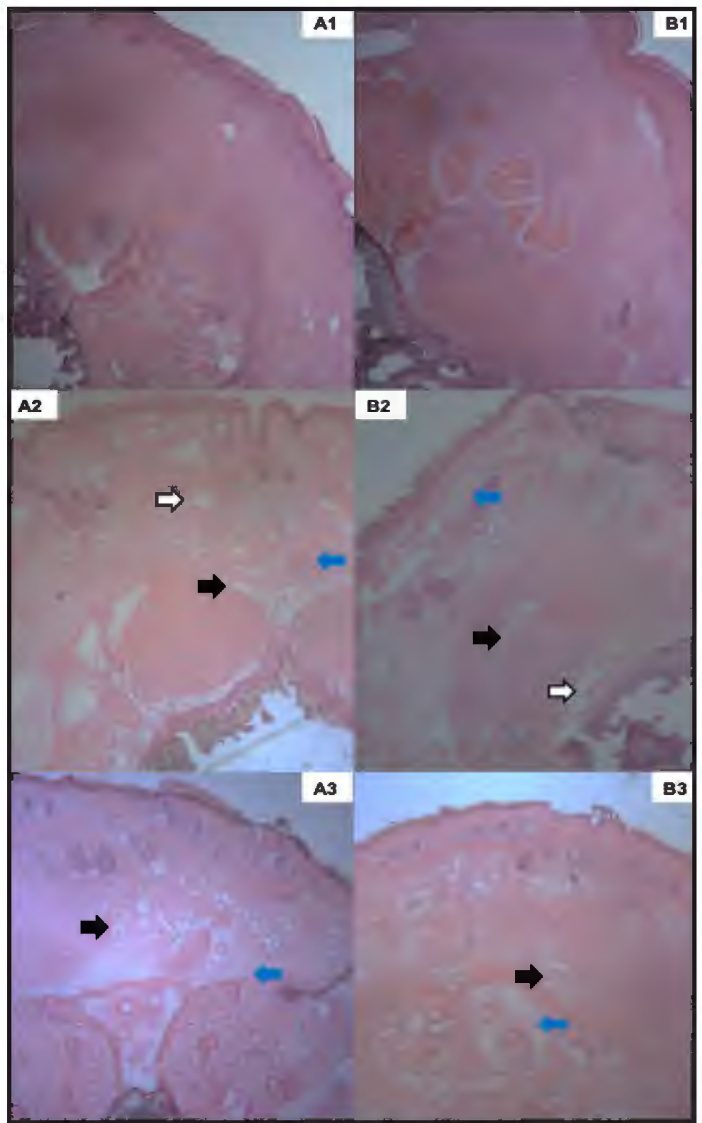

Fig. 5. Panels AI and B1 show the proximal part of the tails (control). Panels A2 and B2 show the distal part of the tails (lymphedema) three weeks after surgical procedure for MODELS A and B, respectively. There is inflammatory cells infiltration and edema (blue arrows), with lymph vessels dilation (white arrows) and a few adipocytes can be seen (black arrows). In panels $A 3$ and $B 3$, we can observe that six weeks after the procedure, the inflammatory process persists (blue arrows) and fat deposition is more prominent (black arrows) in animals operated according to both techniques. Hematoxylin / eosin staining, enlargement = $40 x, A 1, A 2$ and A3 from animals operated according to MODEL $A$ and B1, B2 and B3 from animals operated according to MODEL $B$.

All these findings are mostly due to lymphatic stasis and inflammation. These findings are more prominent six weeks after the surgery with increased deposits of fat tissue and enlargement of lymph vessels (Fig. 5).

Staining with oil-red $\mathrm{O}$, in order to detect adipose tissue, was further performed and a marked increase was observed in tissues ob-

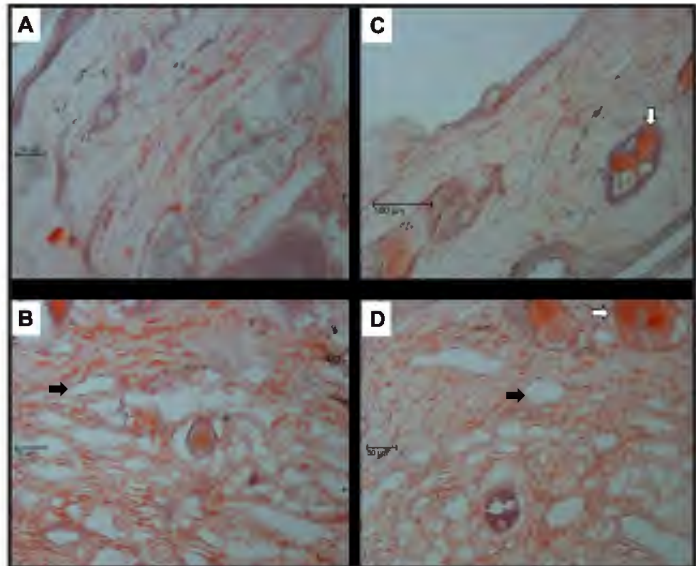

Fig. 6. In order to better characterize adipocytes, Oil Red $O$ staining was performed in samples from animals operated according to MODELS A (Panels A and $B$ ) and B (Panels $C$ and D). Panels $A$ and $C$ show the control group, where fat deposition is observed mainly around the hair follicles (white arrows). Panels $B$ and D, obtained six weeks after surgery according to MODEL A (Panel B) or B (Panel D) demonstrate a marked increase in fat deposition and lymph vessels dilation (black arrows).

tained from the distal part of the tails, where lymphedema was present (Fig. 6).

In both histological methods, no difference could be observed between animals operated according to MODELS A or B. Since these methods are not quantitative, we sought differences between these two models using molecular approaches.

\section{Molecular Markers of Lymphedema Formation}

We further tested mRNA expression of adipose tissue and lymphangiogenesis markers in the proximal and distal areas of the tails in both experimental groups. Lipin 1 and 2 are proteins involved in triglyceride metabolism and are considered markers of adipocyte differentiation (17). A marked increase in mRNA was observed for both lipins 1 and 2 in the lymphedema area as compared to the control portion of the tail (Fig. 7). We also observed a pronounced enhancement in SLP76 mRNA transcription in the lymphedema area compared to their control counterparts. Signaling by SLP-76 is required in circulating cells to 
regulate separation of blood and lymphatic vascular networks (18) (Fig. 7). In addition, we also detected a considerable increase in F4-80 in the lymphedema portion of the tail (Fig. 7). F4-80 is a well-known and widely used marker of murine macrophage populations (19), suggesting that there is a prominent inflammatory reaction occurring in the lymphedema portion of the tail.

Therefore, we conclude that our adapted mouse-tail experimental model is able to induce lymphedema with characteristics of adipose tissue and lymph vessel formation. No differences could be observed on size, morphology, and structure in lymphedema between the model previously described (MODEL A) and the modified version developed in our laboratory (MODEL B).

\section{DISCUSSION}

Secondary lymphedema is a common clinical condition and is frequently observed after radiotherapy or surgical treatment of cancer. Our understanding concerning developmental pathophysiology is still incomplete and better experimental models are needed.

A recent systematic review supported mice as the best experimental model, based on costs, reproducibility, and ease of use. A method for developing lymphedema in mouse tails has been proposed by Boardman and Rutkowski $(15,16)$ and modified by Clavin NW et al (12). This model has the advantage of comparing an area under lymphatic stasis with an area of hypothetical normal physiologic conditions. This could help reduce possible bias related to mechanisms which might

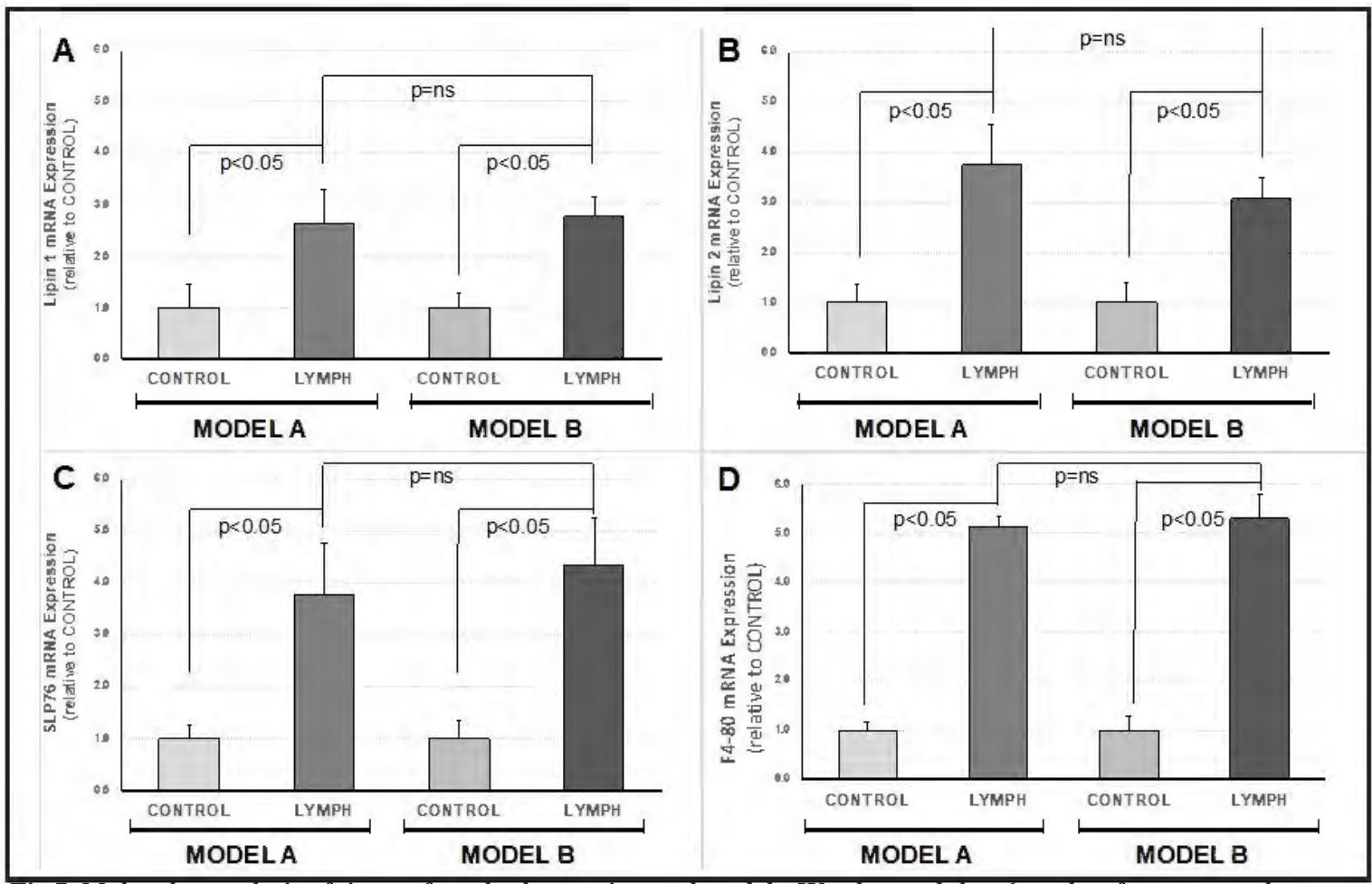

Fig.7. Molecular analysis of tissues from both experimental models. We observed that 6 weeks after surgery there was a marked increase in markers of adipocyte differentiation lipin 1 (panel A) and lipin 2 (Panel B) when compared the distal part of the tail (lymphedema, LYMPH) with its proximal counterpart (CONTROL). However, no difference was observed in comparison between animals operated with MODEL A or MODEL B. Similar pattern was observed in markers for lymphangiogenesis (SLP 76) or macrophage infiltration (F4-80). N = 10 in each group. 
influence inflammation but were not measured in the study, such as body weight, metabolism, hormonal interaction, and immunity status.

In this study, we suggest some modifications of this previously described model. Our adapted experimental model displayed several advantages, compared to other models reported in the literature $(10,12,13-16)$. It is possible to perform this model quickly, since all procedures are performed in less than $\mathbf{3 0}$ minutes, uses inexpensive and accessible materials, and, most importantly, causes no procedure-related mortality and a hundred percent effectiveness in inducing lymphedema. Furthermore, the lateral vein ligation did not cause ischemia, venous insufficiency, infection or tail necrosis in any experimental animals. This is an important finding due to the ligation of two veins in the tail in this modified procedure.

In our model we observed that the mouse tail maintained venous sufficiency as long as one of the three tail veins was kept open. The ligation of the group of structures colored with Patent Blue V stain together with the lateral veins gave more assurance that all lymphatics were blocked and allowed the exclusion of any possible future spontaneous lymphovenous anastomosis which might be induced by lymphatic stasis. This is especially important due to the great proximity between these structures and the evidence of lymphedema disappearance 6 weeks after the procedure in other studies. Moreover, models which involved techniques that could cause potential harm to the lateral veins together with the lymphatic vessels, such as radiotherapy, showed high edema maintenance throughout time. In addition, this long period of development ( 6 weeks) allows the testing of procedures that can reduce lymphedema.

The results of our adapted surgical procedures are very similar to the clinical findings in human lymphedema $(7,8,20)$. There is a marked enlargement distal to the level of lymphatic obstruction, beginning after the procedure and lasting for at least six weeks. Morphology is also very similar with edema and inflammation at the beginning, followed by adipose tissue deposits, as observed in histological analyses (20).

These results were validated by a molecular approach. We observed that the lymphedematous area expressed higher transcription levels of proteins involved in lymphedema formation. We could detect higher levels of mRNA for proteins involved in macrophage infiltration (F4-80), lymph vessel formation (SLP76), and adipocyte differentiation (lipins 1 and 2). One limitation of this work is that we did not examine markers of lymphatics including Prox1, Lyve, prodoplanin, or Vegfr3.

In conclusion, we describe here a new experimental model of lymphedema with several advantages compared to current models in use today. Due to its similarity with the human findings, this experimental model may be useful to gain better understanding of the pathophysiology of lymphedema formation and to evaluate new treatment strategies including the therapeutic window and possible molecular targets to prevent adipose tissue accumulation in lymphedema. Moreover, this model can also be used to study adipogenesis mechanisms and, therefore may provide an opportunity for new understanding and treatments for lipedema and obesity. Replication and further documentation of findings in this model are needed to confirm the reliability and usefulness of this model.

\section{ACKNOWLEDGMENTS}

Research supported by FAPESP (2017/05232-8), CNPq, and CAPES.

\section{CONFLICT OF INTEREST AND DISCLOSURE}

All authors declare no competing financial interests exist.

\section{REFERENCES}

1. Lee, BB, M Andrade, PL Antignani, et al: Diagnosis and treatment of primary lymphedema. Consensus document of the International Union of Phlebology (IUP)-2013. Int. Angiol. 32 (2013), 541-574. 
2. Hayes, SC, M Janda, B Cornish, et al: Lymphedema after breast cancer: incidence, risk factors, and effect on upper body function. $\mathbf{J}$. Clin. Oncol. 26 (2008), 3536-3542.

3. Noguchi, M: Axillary reverse mapping for preventing lymphedema in axillary lymph node dissection and/or sentinel lymph node biopsy. Breast Cancer 17 (2010), 155-157.

4. Finnane, A, M Janda, SC Hayes: Review of the evidence of lymphedema treatment effect. Am. J. Phys. Med. Rehabil. 94 (2015), 483-498.

5. Allen, RJ, MH Cheng: Lymphedema surgery: Patient selection and an overview of surgical techniques. J. Surg. Oncol. 113 (2016), 923-931.

6. Ghanta, S, DA Cuzzone, JS Torrisi, et al: Regulation of inflammation and fibrosis by macrophages in lymphedema. Am. J. Physiol. Heart. Circ. Physiol. 308 (2015), H1065-H1077.

7. Dylke, ES, LC Ward, JD Meerkin, et al: Tissue composition changes and secondary lymphedema. Lymphat. Res. Biol. 11 (2013), 211-218.

8. Brorson, H, K Ohlin, G Olsson, et al: Breast cancer-related chronic arm lymphedema is associated with excess adipose and muscle tissue. Lymphat. Res. Biol. 7 (2009), 3-10.

9. Cuzzone, DA, ES Weitman, NJ Albano, et al: IL-6 regulates adipose deposition and homeostasis in lymphedema. Am. J. Physiol. Heart Circ. Physiol. 306 (2014), H1426-H1434.

10. Hadrian, R, D Palmes: Animal models of secondary lymphedema: New approaches in the search for therapeutic options. Lymphat. Res. Biol 15 (2017), 2-16.

11. Frueh, FS, E Gousopoulos, F Rezaeian, et al: Animal models in surgical lymphedema research--a systematic review. J. Surg. Res. 200 (2016), 208-220.

12. Clavin, NW, T Avraham, J Fernandez, et al: TGF-beta1 is a negative regulator of lymphatic regeneration during wound repair. Am. J. Physiol. Heart Circ. Physiol. 295 (2008), H2113-H2127.
13. Aschen, S, JC Zampell, S Elhadad, et al: Regulation of adipogenesis by lymphatic fluid stasis: part II. Expression of adipose differentiation genes. Plast. Reconstr. Surg. 129 (2012), 838847.

14. Zampell, JC, S Aschen, ES Weitman, et al: Regulation of adipogenesis by lymphatic fluid stasis: part I. Adipogenesis, fibrosis, and inflammation. Plast. Reconstr. Surg. 129 (2012), 825-834.

15. Boardman, KC, MA Swartz: Interstitial flow as a guide for lymphangiogenesis. Circ. Res. 92 (2003), 801-808.

16. Rutkowski, JM, M Moya, J Johannes, et al: Secondary lymphedema in the mouse tail: Lymphatic hyperplasia, VEGF-C upregulation, and the protective role of MMP-9. Microvasc. Res. 72 (2006), 161-171.

17. Csaki, LS, JR Dwyer, LG Fong, et al: Lipins, lipinopathies, and the modulation of cellular lipid storage and signaling. Prog. Lipid Res. 52 (2013), 305-316.

18. Abtahian, F, A Guerriero, E Sebzda, et al: Regulation of blood and lymphatic vascular separation by signaling proteins SLP-76 and Syk. Science 299 (2003), 247-251.

19. Lumeng, CN, JL Bodzin, AR Saltiel: Obesity induces a phenotypic switch in adipose tissue macrophage polarization. J. Clin. Invest. 117 (2007), 175-184.

20. Ly, CL, RP Kataru, BJ Mehrara: Inflammatory Manifestations of Lymphedema. Int. J. Mol. Sci. 18 (2017), 171-183.

Mauro Andrade, MD, PhD

Rua Barata Ribeiro, 237 - CJ. 56

São Paulo, BRAZIL 01308-000

Telephone \#: 55.11.3129.4234

FAX \#: 55.11.3129.4234

E-mail: mauro.andrade@fm.usp.br 